\title{
SOME REMARKS ON THE CONSTRUCTION OF QUANTUM SYMMETRIC SPACES
}

\author{
Mathijs S. DiJkhuizen \\ Department of Mathematics, Faculty of Science, \\ Kobe University, Rokko, Kobe 657, Japan
}

31 May 1995

\begin{abstract}
We present a general survey of some recent developments regarding the construction of compact quantum symmetric spaces and the analysis of their zonal spherical functions in terms of $q$-orthogonal polynomials. In particular, we define a one-parameter family of two-sided coideals in $\mathcal{U}_{q}(\mathfrak{g l}(n, \mathbb{C}))$ and express the zonal spherical functions on the corresponding quantum projective spaces as Askey-Wilson polynomials containing two continuous and one discrete parameter.
\end{abstract}

\section{Introduction}

In this paper, we discuss some recent progress in the analysis of compact quantum symmetric spaces and their zonal spherical functions. It is our aim to present a more or less coherent overview of a number of quantum analogues of symmetric spaces that have recently been studied. We shall try to emphasize those aspects of the theory that distinguish quantum symmetric spaces from their classical counterparts.

It was recognized not long after the introduction of quantum groups by Drinfeld [Dr], Jimbo [J1], and Woronowicz [Wo], that the quantization of symmetric spaces was far from straightforward. We discuss some of the main problems that came up.

First of all, there was the, at first sight rather annoying, lack of interesting quantum subgroups. Let us mention, for instance, the well-known fact that there is no analogue of $S O(n)$ inside the quantum group $S U_{q}(n)$. A major step forward in overcoming this hurdle was made by Koornwinder [K2], who was able to construct a quantum analogue of the classical 2-sphere $S U(2) / S O(2)$ and express the zonal spherical functions as a subclass of Askey-Wilson polynomials by exploiting the notion of a twisted primive element in the quantized universal enveloping algebra $\mathcal{U}_{q}(\mathfrak{s} \mathfrak{l}(2, \mathbb{C}))$. This "infinitesimal" approach was considerably generalized by Noumi

Key words and phrases. compact quantum group, quantum symmetric space, quantum projective space, zonal spherical function, two-sided coideal, Casimir operator, radial part, AskeyWilson polynomials, Macdonald's symmetric polynomials, Koornwinder's $B C$-type Askey-Wilson polynomials.

The author acknowledges financial support by the Japan Society for the Promotion of Science (JSPS) and the Netherlands Organization for Scientific Research (NWO). 
in his fundamental paper [N], where he used the more general notion of a two-sided coideal in $\mathcal{U}_{q}(\mathfrak{g})$ to study quantum analogues of the symmetric spaces $S U(n) / S O(n)$ and $S U(2 n) / S p(n)$ and express their zonal spherical functions as Macdonald's symmetric polynomials associated with root system $A_{n-1}$ (cf. [M1]). In the same paper, both the $L$-operators (cf. [RTF]) and constant solutions of the reflection equation (cf. $[\mathrm{Ku}]$ ) were used for the first time to construct suitable two-sided coideals.

A second rather striking phenomenon was the appearance of parametrized families of quantum symmetric spaces with one and the same classical counterpart. Podleś $[\mathrm{Po}]$ was the first to exhibit a continuously parametrized family of quantum analogues of the classical 2-sphere. The zonal spherical functions on these quantum spheres were completely analysed by Koornwinder [K2], [K4], who used the infinitesimal method.

In this paper, we announce some recent results by M. Noumi and the author showing that the parameter phenomenon is not restricted to 2-spheres but extends to complex projective spaces of arbitrary dimension and even beyond. The zonal spherical functions on our family of quantum projective spaces are expressed as Askey-Wilson polynomials containing two continuous and one discrete parameter.

A third difference is the rather ad hoc approach to quantum symmetric spaces. As far as the author knows, no method has been devised so far that provides a unified approach to even a subclass of quantum symmetric spaces. Related to this are the computational difficulties involved in the quantum case, especially in the computation of the radial part of a quantum Casimir operator. First applied by Koornwinder $[\mathrm{K} 4]$ in the rank one case, then used by Noumi $[\mathrm{N}]$ in the general rank case (see also [UT]), this method has by now become one of the standard means to identify zonal spherical functions on quantum symmetric spaces.

The organization of this paper is as follows. In section 1 we briefly review the theory of classical compact symmetric spaces and their zonal spherical functions. In sections 2 and 3 we recall some basic facts concerning compact quantum groups, particularly the quantum unitary group, and sketch one possible general method to analyse quantum symmetric spaces. In section 4 we announce some recent results by M. Noumi and the author on a family of quantum projective spaces. Finally, in section 5 we briefly discuss some patterns underlying the study of higher rank quantum symmetric spaces.

The author would like to express his sincere gratitude to Prof. M. Noumi and T. Sugitani. Many of the general ideas expressed in this paper originated with them, still others ripened in the countless discussions we had during our informal seminars in Tokyo. The author would also like to thank Prof. Tom H. Koornwinder, who introduced him to the subject of harmonic analysis on quantum groups.

\section{Classical compact symmetric spaces}

The material treated in this section is well-known by now (cf. Helgason [H1], [H2], Heckman and Schlichtkrull [HS]).

Let $G$ be a compact connected simple Lie group. To simplify our statements we assume that $G$ is simply connected, but this is not essential. Let $K \subset G$ be a closed subgroup such that there exists a (necessarily unique) involutive automorphism $\theta: G \rightarrow G$ with $G_{0}^{\theta} \subset K \subset G^{\theta}$. Here $G^{\theta}$ denotes the subgroup of fixed points of $\theta$, $G_{0}^{\theta}$ its connected component of the identity. Then the pair $(G, K)$ is an irreducible 
Riemannian symmetric pair, and the $G$-homogeneous space $G / K$ is an irreducible Riemannian symmetric space of compact type I (in the terminology of Helgason [H1]).

For any (finite-dimensional continuous) irreducible representation $\pi$ of $G$, the trivial representation $\delta$ of $K$ occurs at most once in the irreducible decomposition of the restriction of $\pi$ to $K$. In other words, $(G, K)$ is a Gelfand pair. Those irreducible representations $\pi$ of $G$ that have non-zero $K$-fixed vectors are called spherical representations.

Let $\mathcal{D}(G / K)$ denote the algebra of left $G$-invariant differential operators on the space $G / K$. A (zonal) spherical function is by definition a $K$-biinvariant differentiable function on $G$ which is a joint eigenfunction of all differential operators $D \in \mathcal{D}(G / K)$.

There is a 1-1 correspondence between spherical representations and spherical functions (determined up to a scalar multiple). To make this more explicit, we write $\mathfrak{g} \subset \mathfrak{g}_{\mathbb{C}}$ for the Lie algebra of $G$ and its complexification. Let $\mathfrak{h}_{\mathbb{C}} \subset \mathfrak{g}_{\mathbb{C}}$ be a Cartan subalgebra, $R:=R\left(\mathfrak{g}_{\mathbb{C}}, \mathfrak{h}_{\mathbb{C}}\right)$ the corresponding root system. We fix a choice of positive roots $R^{+} \subset R$. Let $P^{+} \subset P \subset \mathfrak{h}_{\mathbb{C}}^{*}$ denote the corresponding cone $P^{+}$ of dominant weights in the weight lattice $P$. The irreducible representation of $G$ with highest weight $\lambda \in P^{+}$is denoted by $V(\lambda)$. Let $P_{K}^{+} \subset P^{+}$denote the subset of dominant weights corresponding to the spherical representations. If we write $\mathcal{H}$ for the algebra of $K$-biinvariant (continuous) representative (i.e. $G$-finite) functions on $G$, then there is the canonical decomposition

$$
\mathcal{H}=\bigoplus_{\lambda \in P_{K}^{+}} \mathcal{H}(\lambda)
$$

where $\mathcal{H}(\lambda)$ is the intersection of $\mathcal{H}$ and the subspace $W(\lambda) \subset L^{2}(G)$ spanned by the matrix coefficients of the representation $V(\lambda)$. Each of the subspaces $\mathcal{H}(\lambda)$ is one-dimensional. If we choose a non-zero $\varphi_{\lambda} \in \mathcal{H}(\lambda)$, then $\varphi_{\lambda}$ will be a spherical function. The right $G$-translates of $\varphi_{\lambda}$ span a subspace which is equivalent as a representation of $G$ to $V(\lambda)$. All spherical functions can be obtained this way.

In order to give a more explicit description of the spherical functions $\varphi_{\lambda}$, we recall the definition of (generalized) Jacobi polynomials (cf. [HS]). Let $\Sigma \subset V$ be a (possibly non-reduced) root system of rank $l$ in a real vector space $V$. We fix an inner product $\langle\cdot, \cdot\rangle$ on $V$ which is invariant under the Weyl group $W=W_{\Sigma}$. Via this inner product we may identify $V$ with its dual $V^{*}$. We fix a choice $\Sigma^{+} \subset \Sigma$ of positive roots. We then have a cone of dominant weights $P_{\Sigma}^{+}$inside the weight lattice $P_{\Sigma}$. The symbol $\preceq$ denotes the usual dominance ordering of weights. Let $\mathbb{C}\left[P_{\Sigma}\right]$ denote the group algebra of the free abelian group $P_{\Sigma}$ with canonical elements $e^{\lambda} \in \mathbb{C}\left[P_{\Sigma}\right]\left(\lambda \in P_{\Sigma}\right)$. The Weyl group $W$ acts on $\mathbb{C}\left[P_{\Sigma}\right]$ by $w \cdot e^{\lambda}=e^{w \lambda}$. The orbit sums $m_{\lambda}:=\sum_{\mu \in W \lambda} e^{\mu}\left(\lambda \in P_{\Sigma}^{+}\right)$are $W$-invariant and form a basis of the subalgebra $\mathbb{C}\left[P_{\Sigma}\right]^{W}$ of Weyl group invariants. Let $Q^{\vee} \subset V^{*}$ denote the dual root lattice. Then $T:=V^{*} / 2 \pi Q^{\vee}$ is a compact torus. If we interpret $e^{\lambda} \in \mathbb{C}\left[P_{\Sigma}\right]$ as a function on $T$ by $e^{\lambda}(x):=e^{i\langle\lambda, x\rangle}$, then the group algebra $\mathbb{C}\left[P_{\Sigma}\right]$ is identified with the algebra $\mathcal{A}(T)$ of representative functions on the torus $T$. This identification is compatible with the natural actions of $W$ on both algebras.

Let now $k: \Sigma \rightarrow[0, \infty), \alpha \mapsto k_{\alpha}$ be a $W$-invariant (so-called multiplicity) function. We define a continuous weight function on $T$ and the corresponding inner 
product on functions of $T$ :

$$
\delta_{k}(x):=\prod_{\alpha \in \Sigma^{+}}\left|2 \sin \left(\frac{1}{2}\langle\alpha, x\rangle\right)\right|^{2 k_{\alpha}}, \quad\langle f, g\rangle_{k}:=\int_{T} f(x) \overline{g(x)} \delta_{k}(x) \mathrm{d} x
$$

where $\mathrm{d} x$ is the normalized Lebesgue measure on $T$. The Jacobi polynomial $P_{\lambda}^{k} \in$ $\mathbb{C}\left[P_{\Sigma}\right]^{W}$ of degree $\lambda \in P_{\Sigma}^{+}$is defined as the element of the form $\sum_{\mu \preceq \lambda} c_{\lambda, \mu} m_{\mu}$ with $c_{\lambda, \lambda}=1$ such that

$$
\left\langle P_{\lambda}^{k}, m_{\mu}\right\rangle_{k}=0, \quad \mu \in P_{\Sigma}^{+}, \mu \prec \lambda .
$$

This last condition is equivalent to the requirement that $P_{\lambda}^{k}$ satisfies the following second-order partial differential equation:

$$
\left(\Delta+\sum_{\alpha \in \Sigma^{+}} k_{\alpha} \cot \left(\frac{1}{2}\langle\alpha, x\rangle\right) \partial_{\alpha}\right) P_{\lambda}^{k}(x)=-\left\langle\lambda, \lambda+\sum_{\alpha \in \Sigma^{+}} k_{\alpha} \alpha\right\rangle P_{\lambda}^{k}(x) .
$$

Here $\Delta$ is the usual Laplace operator on the Euclidean space $V, \partial_{\alpha}$ the partial derivative in the direction of $\alpha$. We denote the partial differential operator on the left-hand side of (1.3) by $L(k)$. Clearly, the Jacobi polynomials $P_{\lambda}^{k}\left(\lambda \in P_{\Sigma}^{+}\right)$form a basis of the algebra $\mathbb{C}\left[P_{\Sigma}\right]^{W}$. One can prove that

$$
\left\langle P_{\lambda}, P_{\mu}\right\rangle=0, \quad \lambda, \mu \in P_{\Sigma}^{+}, \lambda \neq \mu .
$$

Hence, the $P_{\lambda}^{k}$ are a family of orthogonal polynomials. One can actually show that $L(k)$ is contained in a certain commutative algebra $\mathcal{D}(k)$ of self-adjoint differential operators generated by $l$ algebraically independent generators. The $P_{\lambda}^{k}$ can be characterized as the joint eigenfunctions in $\mathbb{C}\left[P_{\Sigma}\right]^{W}$ of the operators in $\mathcal{D}(k)$.

Let us now return to the setting of the symmetric space $G / K$. Let $\mathfrak{k} \subset \mathfrak{g}$ denote the Lie algebra of $K$. The eigenspace decomposition of $\theta: \mathfrak{g} \rightarrow \mathfrak{g}$ is written as $\mathfrak{g}=\mathfrak{k} \oplus i \mathfrak{p} \subset \mathfrak{g}_{\mathbb{C}}$. Let $\mathfrak{a} \subset \mathfrak{p}$ be a maximal abelian subspace. Write $\Sigma^{\prime} \subset \mathfrak{a}^{*}$ for the restricted root system of $G / K, m_{\alpha}$ for the multiplicity of $\alpha \in \Sigma$.

Let us put

$$
\Sigma:=2 \Sigma^{\prime}, \quad k_{2 \alpha}:=\frac{1}{2} m_{\alpha} .
$$

Then $k: \alpha \mapsto k_{\alpha}$ is a $W$-invariant multiplicity function on the root system $\Sigma \subset \mathfrak{a}^{*}$.

Now $T:=\exp (i \mathfrak{a}) / \exp (i \mathfrak{a}) \cap K$ is a compact torus in $G$ isomorphic with the quotient $\mathfrak{a} / 2 \pi Q_{\Sigma}^{\vee}$ via the exponential mapping. Restriction defines an algebra isomorphism of $\mathcal{H}$ onto the algebra $\mathcal{A}(T)^{W}$ of $W$-invariant representative functions on the torus $T$.

Let $D \in \mathcal{D}(G / K)$ be a $G$-invariant differential operator on $G / K$. It is obvious that $D$ maps $\mathcal{H}$ into itself. There is a uniquely determined differential operator $\operatorname{rad}(D)$ acting on functions on $T$ such that $\operatorname{rad}(D)\left(f_{\mid T}\right)=D(f)$. This differential operator is called the radial part of $D$. The mapping rad actually induces an algebra isomorphism of $\mathcal{D}(G / K)$ onto the algebra $\mathcal{D}(k)$, where the multiplicity function $k$ is defined in (1.4). Under this isomorphism, the Laplace-Beltrami operator $\Delta$ on the Riemannian symmetric space $G / K$ is mapped onto the operator $L(k)$. Let us recall that $\Delta$ arises as the image of the standard Casimir operator on $G$ which can be viewed as a central element of the universal enveloping algebra $\mathcal{U}\left(\mathfrak{g}_{\mathbb{C}}\right)$. 
Suppose now that the Cartan subalgebra $\mathfrak{h}_{\mathbb{C}} \subset \mathfrak{g}_{\mathbb{C}}$ is invariant under the complexlinear extension of $\theta$ to $\mathfrak{g}_{\mathbb{C}}$, and that, in addition, $\mathfrak{a} \subset \mathfrak{h}_{\mathbb{C}}$. Then we have the decomposition $\mathfrak{h}_{\mathbb{C}}=\mathfrak{a}_{\mathbb{C}} \oplus\left(\mathfrak{k}_{\mathbb{C}} \cap \mathfrak{h}_{\mathbb{C}}\right)$. The representation $V(\lambda)\left(\lambda \in P^{+}\right)$is spherical if and only if $\lambda$ vanishes on $\mathfrak{k}_{\mathbb{C}} \cap \mathfrak{h}_{\mathbb{C}}$ and the restriction $\mu$ of $\lambda$ to $\mathfrak{a}$ is a dominant weight in $P_{\Sigma}^{+}$. The spherical function $\varphi_{\lambda}\left(\lambda \in P_{K}^{+}\right)$, when restricted to $T$, is a scalar multiple of the Jacobi polynomial $P_{\mu}^{k}$, where $k$ is defined as in (1.4).

Since the spherical functions $\varphi_{\lambda}$ are matrix coefficients of irreducible representations, the Schur orthogonality relations imply that the $\varphi(\lambda)$ are mutually orthogonal with respect to the inner product on $L^{2}(G)$. It can be shown that this inner product coincides on $\mathcal{H}$ with the inner product (1.2) up to a scalar multiple.

\section{Compact quantum groups}

In the remainder of this paper, we assume that $G$ is one of the following compact Lie groups: $U(n), S U(n)(n \geq 2), S O(n)(n \geq 3), S p(n)(n \geq 2)$. Actually, to simplify our statements, we take $G=U(n)$ in this section. Mutatis mutandis all the definitions and statements in this section are valid for the cases $S U(n), S O(n)$, and $S p(n)$ too.

Let us fix $0<q<1$ and $n \geq 2$. We identify $\mathfrak{g}_{\mathbb{C}}$ with the Lie algebra $\mathfrak{g l}(n, \mathbb{C})$ of complex $n \times n$ matrices. As Cartan subalgebra $\mathfrak{h}_{\mathbb{C}} \subset \mathfrak{g}_{\mathbb{C}}$ we take the subspace of diagonal matrices. Then, with the usual choice of $\varepsilon_{i} \in \mathfrak{h}_{\mathbb{C}}^{*}$, we can identify the weight lattice $P$ with the free $\mathbb{Z}$-span of the $\varepsilon_{i}$. There is a unique $\mathbb{Z}$-valued symmetric bilinear form $\langle\cdot, \cdot\rangle$ on $P$ such that $\left\langle\varepsilon_{i}, \varepsilon_{j}\right\rangle=\delta_{i j}$. Via this pairing, we shall identify $P$ with its dual $P^{*}=\operatorname{Hom}_{\mathbb{Z}}(P, \mathbb{Z})$. We put $\alpha_{i}:=\varepsilon_{i}-\varepsilon_{i+1}(1 \leq i \leq n-1)$.

The quantized universal enveloping algebra $\mathcal{U}_{q}=\mathcal{U}_{q}(\mathfrak{g r}(n, \mathbb{C}))(\mathrm{cf} .[\mathrm{Dr}],[\mathrm{J} 1],[\mathrm{J} 2]$, $[\mathrm{N}])$ is the algebra generated by the symbols $q^{h}\left(h \in P^{*}\right)$ and $e_{i}, f_{i}(1 \leq i \leq n-1)$ subject to the following relations:

$$
\begin{aligned}
& q^{0}=1, \quad q^{h+h^{\prime}}=q^{h} \cdot q^{h^{\prime}}, \\
& q^{h} e_{i} q^{-h}=q^{\left\langle h, \alpha_{i}\right\rangle} e_{i}, \quad q^{h} f_{i} q^{-h}=q^{-\left\langle h, \alpha_{i}\right\rangle} f_{i}, \\
& e_{i} f_{j}-f_{j} e_{i}=\delta_{i j} \frac{q^{\alpha_{i}}-q^{-\alpha_{i}}}{q-q^{-1}}, \\
& e_{i}^{2} e_{j}-\left(q+q^{-1}\right) e_{i} e_{j} e_{i}+e_{j} e_{i}^{2}=0(|i-j|=1) ; \quad e_{i} e_{j}=e_{j} e_{i}(|i-j|>1), \\
& f_{i}^{2} f_{j}-\left(q+q^{-1}\right) f_{i} f_{j} f_{i}+f_{j} f_{i}^{2}=0(|i-j|=1) ; \quad f_{i} f_{j}=f_{j} f_{i}(|i-j|>1),
\end{aligned}
$$

where $h, h^{\prime} \in P^{*}$ and $1 \leq i, j \leq n-1$.

Let $\mathcal{U}_{q}(\mathfrak{h}) \subset \mathcal{U}_{q}$ denote the subalgebra generated by the elements $q^{h}\left(h \in P^{*}\right)$. It is a Laurent polynomial algebra in the generators $q^{\varepsilon_{i}}$.

A left $\mathcal{U}_{q}$-module $W$ is called $P$-weighted if it has a vector space basis consisting of weight vectors with weights in $P$. The action of $\mathcal{U}_{q}(\mathfrak{h})$ on any $P$-weighted $\mathcal{U}_{q^{-}}$ module is diagonalizable, and any such module is completely reducible (cf. [R], [Lz]). The cone of dominant weights $P^{+} \subset P$ consists of all weights $\lambda=\sum_{k} \lambda_{k} \varepsilon_{k} \in P$ such that $\lambda_{1} \geq \ldots \geq \lambda_{n}$. There is a 1-1 correspondence $\lambda \longleftrightarrow V(\lambda)$ between dominant weights and irreducible $P$-weighted finite-dimensional $\mathcal{U}_{q}$-modules such that $\lambda \in P^{+}$is the highest weight of $V(\lambda)$ (cf. [R], [Lz]). Recall that $\lambda \in P^{+}$is called a highest weight of an irreducible $\mathcal{U}_{q}$-module $V$ if there exists a non-zero vector $v \in V$ such that $q^{h} \cdot v=q^{\langle h, \lambda\rangle} v$ and $e_{i} \cdot v=0$ for all $1 \leq i \leq n-1$. 
We denote by $L_{i j}^{+}, L_{i j}^{-}$the so-called $L$-operators introduced in [RTF]. For a precise definition of these operators in the present context see [N]. One has $L_{i j}^{+}=L_{j i}^{-}=0$ $(i>j)$. Moreover,

$$
q^{h} L_{i j}^{ \pm} q^{-h}=q^{\left\langle h, \varepsilon_{j}-\varepsilon_{i}\right\rangle} L_{i j}^{ \pm}, \quad q^{h} S\left(L_{i j}^{ \pm}\right) q^{-h}=q^{\left\langle h, \varepsilon_{j}-\varepsilon_{i}\right\rangle} S\left(L_{i j}^{ \pm}\right),
$$

for $1 \leq i, j \leq n$. Because of this property, the $L_{i j}^{ \pm}$can be viewed as $q$-analogues of the root vectors in $\mathfrak{g}_{\mathbb{C}}=\mathfrak{g l}(n, \mathbb{C})$.

The $L_{i j}^{ \pm}$generate the algebra $\mathcal{U}_{q}$. There is a unique Hopf $*$-algebra structure on $\mathcal{U}_{q}$ such that

$$
\Delta\left(L_{i j}^{ \pm}\right)=\sum_{k} L_{i k}^{ \pm} \otimes L_{k j}^{ \pm}, \quad \varepsilon\left(L_{i j}^{ \pm}\right)=\delta_{i j}, \quad\left(L_{i j}^{ \pm}\right)^{*}=S\left(L_{j i}^{\mp}\right),
$$

for $1 \leq i, j \leq n$. The subalgebra $\mathcal{U}_{q}(\mathfrak{h}) \subset \mathcal{U}_{q}$ is a Hopf $*$-subalgebra. All $P$-weighted finite-dimensional representations of $\mathcal{U}_{q}$ are unitarizable.

Let $\rho_{V}: \mathcal{U}_{q} \rightarrow \operatorname{End}(V)$ denote the irreducible representation (called vector representation) of $\mathcal{U}_{q}$ with highest weight $\varepsilon_{1} \in P^{+}$. Put $N:=\operatorname{dim}(V)$ (in the case $G=U(n)$, one actually has $N=n)$. Fixing a suitable basis $\left(v_{i}\right)$ of $V$ such that $\mathcal{U}_{q}(\mathfrak{h})$ acts by diagonal matrices, one has

$$
R^{ \pm}=\sum_{i j} e_{i j} \otimes \rho_{V}\left(L_{i j}^{ \pm}\right) \in \operatorname{End}(V \otimes V)
$$

where $R^{ \pm} \in \operatorname{End}(V \otimes V)$ (cf. [J3], [RTF], [N]) are the invertible $N^{2} \times N^{2}$ matrices defined by

$$
R:=\sum_{i j} q^{\delta_{i j}} e_{i i} \otimes e_{j j}+\left(q-q^{-1}\right) \sum_{i>j} e_{i j} \otimes e_{j i}, \quad R^{+}:=P R P, \quad R^{-}:=R^{-1} .
$$

Here the $e_{i j} \in \operatorname{End}(V)$ are the standard unit matrices with respect to the basis $\left(v_{i}\right)$, and $P \in \operatorname{End}(V \otimes V)$ is the usual permutation operator.

Denote by $\operatorname{det}_{q}^{-1}: \mathcal{U}_{q} \rightarrow \mathbb{C}$ the one-dimensional representation with highest weight $\lambda=-\varepsilon_{1}-\cdots-\varepsilon_{n}$. The algebra $\mathcal{A}_{q}=\mathcal{A}_{q}(U(n))$ (cf. [RTF], [N]) of representative functions on the quantum unitary group $U_{q}(n)$ is the subalgebra of the linear dual of $\mathcal{U}_{q}$ generated by $\operatorname{det}_{q}^{-1}$ and the coefficients $\left(t_{i j}\right)$ of the vector representation $V$ with respect to the basis $\left(v_{i}\right)$. The generators $t_{i j}$ and $\operatorname{det}_{q}^{-1}$ satisfy the relations

$$
R T_{1} T_{2}=T_{2} T_{1} R, \quad \operatorname{det}_{q} \cdot \operatorname{det}_{q}^{-1}=\operatorname{det}_{q}^{-1} \cdot \operatorname{det}_{q} .
$$

Here $T:=\left(t_{i j}\right)_{1 \leq i, j \leq n}$ is an $N \times N$ matrix with coefficients in $\mathcal{A}_{q}, T_{1}:=T \otimes$ id and $T_{2}:=\mathrm{id} \otimes T$ are Kronecker matrix products, and $\operatorname{det}_{q} \in \mathcal{A}_{q}$ (called the quantum determinant) is defined as

$$
\operatorname{det}_{q}:=\sum_{w \in \mathfrak{S}_{n}}(-q)^{l(w)} t_{w(1) 1} \cdots t_{w(n) n} .
$$

Here $\mathfrak{S}_{n}$ denotes the permutation group on $n$ letters, and $l(w)$ denotes the length of a permutation $w \in \mathfrak{S}_{n}$. The elements $\operatorname{det}_{q}$ and $\operatorname{det}_{q}^{-1}$ are central in $\mathcal{A}_{q}$. 
There is a unique Hopf $*$-algebra structure on $\mathcal{A}_{q}$ such that

$$
\Delta\left(t_{i j}\right)=\sum_{k} t_{i k} \otimes t_{k j}, \quad \varepsilon\left(t_{i j}\right)=\delta_{i j}, \quad t_{i j}^{*}=S\left(t_{j i}\right),
$$

for all $1 \leq i, j \leq n$, and

$$
\Delta\left(\operatorname{det}_{q}\right)=\operatorname{det}_{q} \otimes \operatorname{det}_{q}, \quad \varepsilon\left(\operatorname{det}_{q}\right)=1, \quad\left(\operatorname{det}_{q}\right)^{*}=S\left(\operatorname{det}_{q}\right)=\operatorname{det}_{q}^{-1} .
$$

The Hopf $*$-algebra $\mathcal{A}_{q}$ is by definition spanned by the coefficients of its finitedimensional unitary corepresentations (cf. [Wo], [DK2]). Any $P$-weighted representation of $\mathcal{U}_{q}$ can be lifted to a corepresentation of $\mathcal{A}_{q}$.

Using the duality $\langle\cdot, \cdot\rangle$ between $\mathcal{U}_{q}$ and $\mathcal{A}_{q}$, one defines a $\mathcal{U}_{q}$-bimodule structure on $\mathcal{A}_{q}$ by putting:

$$
u \cdot a:=(\mathrm{id} \otimes u) \circ \Delta(a), \quad a \cdot u:=(u \otimes \mathrm{id}) \circ \Delta(a), \quad u \in \mathcal{U}_{q}, a \in \mathcal{A}_{q} .
$$

Here, on the right-hand side of both equalities, $u \in \mathcal{U}_{q}$ is viewed as a linear form on $\mathcal{A}_{q}$ via the pairing $\langle\cdot, \cdot\rangle$. The multiplication $\mathcal{A}_{q} \otimes \mathcal{A}_{q} \rightarrow \mathcal{A}_{q}$ and the unit mapping $\mathbb{C} \rightarrow \mathcal{A}_{q}$ then become $\mathcal{U}_{q}$-bimodule homomorphisms. In other words, $\mathcal{A}_{q}$ is an algebra with two-sided $\mathcal{U}_{q}$-symmetry.

Let $W(\lambda) \subset \mathcal{A}_{q}\left(\lambda \in P^{+}\right)$denote the subspace spanned by the coefficients of the (co-)representation $V(\lambda)$. Then one has the following multiplicity-free decomposition of the $\mathcal{U}_{q}$-bimodule $\mathcal{A}_{q}$ into irreducible constituents:

$$
\mathcal{A}_{q}=\bigoplus_{\lambda \in P^{+}} W(\lambda), \quad W(\lambda) \simeq V(\lambda)^{*} \otimes V(\lambda) .
$$

The above decomposition can also be characterized as the simultaneous eigenspace decomposition under the (left) action on $\mathcal{A}_{q}$ of the center $\mathcal{Z} \mathcal{U}_{q} \subset \mathcal{U}_{q}$.

Let $h: \mathcal{A}_{q} \rightarrow \mathbb{C}$ denote the normalized Haar functional (cf. [Wo], [DK2]) on the compact quantum group $G_{q}$. By putting $\langle a, b\rangle=h\left(b^{*} a\right)$ we define a positive definite Hermitian form $\langle\cdot, \cdot\rangle$ on $\mathcal{A}_{q}$. The subspaces $W(\lambda)$ are mutually orthogonal with respect to this inner product.

There is one particularly important subgroup inside the quantum group $G_{q}$. Let $\mathcal{A}(\mathbb{T}):=\mathbb{C}\left[z_{1}, \ldots, z_{n}, z_{1}^{-1}, \ldots, z_{n}^{-1}\right]$ be the algebra of Laurent polynomials in the variables $z_{i}(1 \leq i \leq n)$. We use the notation $z^{\lambda}:=z_{1}^{\left\langle\lambda, \varepsilon_{1}\right\rangle} \cdots z_{n}^{\left\langle\lambda, \varepsilon_{n}\right\rangle}(\lambda \in P)$. There is a unique Hopf $*$-algebra structure on $\mathcal{A}(\mathbb{T})$ such that

$$
\Delta\left(z_{i}\right)=z_{i} \otimes z_{i}, \quad \varepsilon\left(z_{i}\right)=1, \quad z_{i}^{*}=z_{i}^{-1} \quad(1 \leq i \leq n) .
$$

The subalgebra $\mathcal{U}_{q}(\mathfrak{h})$ naturally is in Hopf $*$-algebra duality with $\mathcal{A}(\mathbb{T})$ via

$$
\left\langle q^{h}, z^{\lambda}\right\rangle:=q^{\langle h, \lambda\rangle} .
$$

There is a unique surjective Hopf $*$-algebra morphism

$$
{ }_{\mid \mathbb{T}}: \mathcal{A}_{q} \longrightarrow \mathcal{A}(\mathbb{T})
$$

mapping $t_{i j} \in \mathcal{A}_{q}$ onto $\delta_{i j} z_{i} \in \mathcal{A}(\mathbb{T})$ and $\operatorname{det}_{q} \in \mathcal{A}_{q}$ onto $z_{1} \cdots z_{n} \in \mathcal{A}(\mathbb{T})$. The torus $\mathbb{T}$ can be viewed as a maximal toral subgroup of the quantum group $G_{q}$. The mapping $\mid \mathbb{T}$ then is the corresponding restriction of functions. The mappings $\mid \mathbb{T}$ and $\mathcal{U}_{q}(\mathfrak{h}) \hookrightarrow \mathcal{U}_{q}$ are dual to each other.

In the classical case, one can freely move around the maximal torus $\mathbb{T} \subset G$ by conjugation. After quantization, however, this is no longer true. Since the group of Hopf algebra automorphisms of $\mathcal{A}_{q}(G)$ is rather small (cf. [Ch]), there is very little possibility for changing the position of $\mathbb{T}$ inside the quantum group $G_{q}$. This fact will prove to be of cardinal importance in the analysis of quantum symmetric spaces. 


\section{Quantum homogeneous spaces}

We recall some general facts (cf. [DK1]). The "infinitesimal" method to construct quantum homogeneous spaces uses the idea that the algebra of functions on the homogeneous space $G / K$ can also be defined as the subspace of those functions on $G$ which are annihilated by the $G$-invariant differential operators $X \in \mathfrak{k}_{\mathbb{C}} \subset \mathfrak{g}_{\mathbb{C}}$.

In fact, suppose that $\mathfrak{k}_{q} \subset \mathcal{U}_{q}$ is a two-sided coideal invariant under the mapping $\tau=* \circ S: \mathcal{U}_{q} \rightarrow \mathcal{U}_{q}$. Recall that a subspace $\mathfrak{k}_{q} \subset \mathcal{U}_{q}$ is called a two-sided coideal if $\Delta\left(\mathfrak{k}_{q}\right) \subset \mathfrak{k}_{q} \otimes \mathcal{U}_{q}+\mathcal{U}_{q} \otimes \mathfrak{k}_{q}$ and $\varepsilon\left(\mathfrak{k}_{q}\right)=0$. Then the subspace

$$
B_{\mathfrak{k}_{q}}:=\left\{a \in \mathcal{A}_{q} \mid a \cdot \mathfrak{k}_{q}=0\right\}
$$

of right $\mathfrak{k}_{q}$-invariant functions is a $*$-subalgebra and right coideal in $\mathcal{A}_{q}$, and a left $\mathcal{U}_{q}$-submodule.

Suppose we have a surjective Hopf $*$-algebra morphism $\pi: \mathcal{A}_{q}(G) \rightarrow \mathcal{A}_{q}(K)$ and a dual Hopf $*$-algebra mapping $\psi: \mathcal{U}_{q}\left(\mathfrak{k}_{\mathbb{C}}\right) \rightarrow \mathcal{U}_{q}\left(\mathfrak{g}_{\mathbb{C}}\right)$. Then the subspace $\mathfrak{k}_{q} \subset$ $\mathcal{U}_{q}\left(\mathfrak{g}_{\mathbb{C}}\right)$ spanned by the elements $\psi(v)-\varepsilon(v) 1\left(v \in \mathcal{U}_{q}\left(\mathfrak{k}_{\mathbb{C}}\right)\right)$ is a $\tau$-invariant coideal. Left or right invariance with respect to the quantum subgroup $K_{q} \hookrightarrow G_{q}$ then is the same as invariance with respect to $\mathfrak{k}_{q}$. Unfortunately, as is well-known, the quantization procedure as given by Drinfeld and Jimbo is not functorial (cf. [Ha]). In other words, to an embedding of compact Lie groups $K \hookrightarrow G$ there need not correspond a surjective Hopf $*$-algebra morphism $\mathcal{A}_{q}(G) \rightarrow \mathcal{A}_{q}(K)$. Actually, the supply of Riemannian symmetric pairs $(G, K)$ whose embedding $K \hookrightarrow G$ survives quantization turns out to be rather limited (cf. section 5 ).

Suppose we have a $\tau$-invariant two-sided coideal $\mathfrak{k}_{q} \subset \mathcal{U}_{q}$. Then the pair $\left(\mathcal{U}_{q}, \mathfrak{k}_{q}\right)$ is called a quantum Gelfand pair if, for any finite-dimensional irreducible $P$-weighted representation $V$ of $\mathcal{U}_{q}$, the subspace $V_{\mathfrak{k}_{q}}:=\left\{v \in V \mid \mathfrak{k}_{q} \cdot v=0\right\}$ of so-called $\mathfrak{k}_{q}$-fixed vectors is at most one-dimensional. Those representations $V$ for which the subspace $V_{\mathfrak{k}_{q}}$ is non-zero are called spherical. Let us denote the corresponding subset of dominant weights by $P_{\mathfrak{k}_{q}}^{+}$. Given a quantum Gelfand pair $\left(\mathcal{U}_{q}, \mathfrak{k}_{q}\right)$ one can define the $*$-algebra $\mathcal{H}$ of $\mathfrak{k}_{q}$-biinvariant functions as

$$
\mathcal{H}:=\left\{a \in \mathcal{A}_{q} \mid a \cdot \mathfrak{k}_{q}=0 \text { and } \mathfrak{k}_{q} \cdot a=0\right\} .
$$

Once again, one has a canonical decomposition $\mathcal{H}=\bigoplus_{\lambda \in P_{\mathbf{t}_{q}}^{+}} \mathcal{H}(\lambda)$, where $\mathcal{H}(\lambda)$ is the intersection of $\mathcal{H}$ with the subspace $W(\lambda) \subset \mathcal{A}_{q}$. Each of the subspaces $\mathcal{H}(\lambda)$ is one-dimensional. One can now define a (zonal) spherical function as a non-zero element of $\mathcal{H}(\lambda)\left(\lambda \in P_{\mathfrak{k}_{q}}^{+}\right)$. Spherical functions corresponding to different $\lambda \in P_{\mathfrak{k}_{q}}^{+}$ will be orthogonal with respect to the inner product on $\mathcal{A}_{q}$ defined in terms of the Haar functional $h: \mathcal{A}_{q} \rightarrow \mathbb{C}$.

Suppose now that the restriction of the mapping ${ }_{\mid \mathbb{T}}: \mathcal{A}_{q} \rightarrow \mathcal{A}(\mathbb{T})$ to $\mathcal{H}$ is injective onto its image $\mathcal{H}_{\mid \mathbb{T}}$ (as we will see, there are interesting cases in which this condition is not fulfilled). The algebra $\mathcal{H}$ will be commutative then. Let $C \in \mathcal{Z U}_{q}$ be a suitable central element (Casimir operator). The left action of $C$ on $\mathcal{A}_{q}$ will preserve the subalgebra $\mathcal{H}$. Hence, there is a uniquely determined operator $D: \mathcal{H}_{\mid \mathbb{T}} \rightarrow \mathcal{H}_{\mid \mathbb{T}}$ such that on $\mathcal{H}$ we have

$$
|\mathbb{T} \circ C=D \circ| \mathbb{T},
$$

where the symbol $C$ denotes the left action of $C \in \mathcal{U}_{q}$ on the subalgebra $\mathcal{H}$. The operator $D$ will be called the radial part of the Casimir operator $C$. Since $C$ is 
central, it acts as a scalar on every subspace $\mathcal{H}(\lambda)\left(\lambda \in P_{\mathfrak{k}}^{+}\right)$. In other words, the restriction $\varphi(\lambda)_{\mid \mathbb{T}}$ of the spherical function $\varphi(\lambda) \in \mathcal{H}(\lambda)\left(\lambda \in P_{\mathfrak{k}}^{+}\right)$to the maximal torus $\mathbb{T}$ is an eigenfunction of the operator $D$. If we are able to compute an explicit expression for the operator $D$, this is likely to give us a strong clue to the nature of the spherical function $\varphi(\lambda)$.

Summarizing, we can say that one possible approach to tackling the problem of quantizing the symmetric pair $(G, K)$ and describing the corresponding $q$-spherical functions consists of the following steps.

(i) Find an appropriate $\tau$-invariant coideal $\mathfrak{k}_{q} \subset \mathcal{U}_{q}$ which is a $q$-analogue of the Lie algebra $\mathfrak{k}_{\mathbb{C}}$ of $K$. Show that the pair $\left(\mathcal{U}_{q}, \mathfrak{k}_{q}\right)$ is a quantum Gelfand pair and that the spherical representations are indexed by the same subset of highest weights as in the classical case.

(ii) Investigate whether $\mathfrak{k}_{q}$-biinvariant functions on $G_{q}$ are completely determined by their restriction to the maximal torus $\mathbb{T} \subset G_{q}$, and, if so, give an explicit description of the image $\mathcal{H}_{\mid \mathbb{T}}$ of $\mathcal{H}$ under the restriction mapping $\mid \mathrm{T}$.

(iii) Find an explicit expression for the radial part $D$ of some suitable Casimir element $C \in \mathcal{Z U}_{q}$.

\section{Quantum projective spaces}

In this section we carry out the programme sketched at the end of section 3 for the case of a complex projective space. The results in this section are joint work with M. Noumi. For proofs and more details the reader is referred to the forthcoming paper $[\mathrm{DN}]$.

We briefly review the classical case to provide motivation for what follows. Set $G=U(n), K=U(n-1) \times U(1) \subset U(n)$. The corresponding involution $\theta: \mathfrak{g}_{\mathbb{C}} \rightarrow \mathfrak{g}_{\mathbb{C}}$ is given by $\theta(X)=J X J^{-1}$ with

$$
J=\operatorname{diag}\{1, \ldots, 1,-1\} .
$$

One has $P_{K}^{+}=\left\{l\left(\varepsilon_{1}-\varepsilon_{n}\right) \in P \mid l \in \mathbb{Z}_{+}\right\}$. The Lie subalgebra $\mathfrak{k}_{\mathbb{C}}$ is equal to $\mathfrak{g l}(n-1, \mathbb{C}) \oplus \mathfrak{g l}(1, \mathbb{C})$. The maximal abelian subspace $\mathfrak{a}_{\mathbb{C}} \subset \mathfrak{p}_{\mathbb{C}}$ is the one-dimensional subspace spanned by the matrix $X=e_{1 n}+e_{n 1}$. The restricted root system is isomorphic with $B C_{1}$. The root multiplicities are 1 (long roots) and $2(n-2)$ (short roots).

Let us recall that the (classical) Jacobi poynomials $P_{n}^{(\alpha, \beta)}(x)$ are one-variable polynomials which are orthogonal on the interval $[-1,1]$ with respect to the continuous weight function $w(x)=(1-x)^{\alpha}(1+x)^{\beta}(\alpha, \beta>-1)$. They actually coincide with the generalized Jacobi polynomials corresponding to the root system $B C_{1}$ (there is a simple linear relation between the parameters $\alpha, \beta$, and the values of the multiplicity function $k$ ). The usual Casimir operator $C \in \mathcal{U}\left(\mathfrak{g}_{\mathbb{C}}\right)$ induces a $G$-invariant differential operator on $G / K$ whose radial part essentially coincides with the differential operator in (1.3) for the values $\alpha=n-2, \beta=0$. Hence, the spherical function $\varphi_{l}$ corresponding to the highest weight $l\left(\varepsilon_{1}-\varepsilon_{n}\right)\left(l \in \mathbb{Z}_{+}\right)$can be expressed as a Jacobi polynomial $P_{l}^{(n-2,0)}$.

Our choice of the involution $\theta$ is such that the corresponding maximal abelian subspace $\mathfrak{a}_{\mathbb{C}}$ does not contain any diagonal matrices. Hence the restriction of $K$ biinvariant functions to the diagonal subgroup $\mathbb{T}$ is hardly injective. To remedy this 
situation, one could take a different choice of involution, for instance by putting

$$
J^{\prime}:=\operatorname{diag}(0,1, \ldots, 1,0)-e_{n 1}-e_{n 1},
$$

and defining $\theta^{\prime}(X)=J^{\prime} X J^{\prime-1}$. The corresponding $\mathfrak{a}_{\mathbb{C}}^{\prime}$ is spanned by the matrix $X^{\prime}=e_{11}-e_{n n}$ and hence is contained in the Cartan subalgebra $\mathfrak{h}_{\mathbb{C}}$. With this choice of $\theta^{\prime}$ and the corresponding subgroup $K^{\prime} \subset G$, the restriction mapping $\mathcal{H}^{\prime}=\mathcal{A}\left(K^{\prime} \backslash G / K^{\prime}\right) \rightarrow \mathcal{A}(\mathbb{T})$ actually is injective. Of course, the symmetric spaces $G / K$ and $G / K^{\prime}$ are isometric and their spherical functions essentially the same, since the involutions $\theta$ and $\theta^{\prime}$ differ only by a conjugation. Alternatively, one could have moved the maximal torus $\mathbb{T}$ inside $G$ so as to make the restriction of $K$-biinvariant functions injective.

We now turn to the quantum case. Let us fix real numbers $c, d \geq 0$ such that $(c, d) \neq(0,0)$. The subspace $\mathfrak{k}^{(c, d)} \subset \mathcal{U}_{q}$ is by definition spanned by the following elements:

$$
\begin{aligned}
& \text { (i) } L_{11}^{+}-L_{n n}^{-}, L_{11}^{-}-L_{n n}^{+}, \\
& \text {(ii) } \sqrt{c} L_{1 k}^{+}+\sqrt{d} L_{n k}^{-} \quad(2 \leq k \leq n-1), \\
& \text { (iii) } \sqrt{d} L_{k n}^{+}+\sqrt{c} L_{k 1}^{-} \quad(2 \leq k \leq n-1), \\
& \text { (iv) } L_{i j}^{+}, L_{j i}^{-} \quad(2 \leq i<j \leq n-1), \\
& \text { (v) } L_{i i}^{+}-L_{i i}^{-} \quad(2 \leq i \leq n-1), \\
& \text { (vi) } \sqrt{c d} L_{1 n}^{+}-\sqrt{c d} L_{n 1}^{-}-(c-d)\left(L_{11}^{+}-L_{11}^{-}\right) .
\end{aligned}
$$

We remark that the subspace $\mathfrak{k}^{(c, d)} \subset \mathcal{U}_{q}$ only depends on the ratio of the numbers $c$ and $d$. In fact, given $c, d \geq 0$ such that $(c, d) \neq(0,0)$, we can define $\sigma \in \mathbb{R} \cup\{ \pm \infty\}$ by $q^{\sigma}=\sqrt{\frac{d}{c}}(c, d>0), \sigma=-\infty(c=0), \sigma=\infty(d=0)$. Then the coideal $\mathfrak{k}^{(c, d)}$ only depends on the value of the corresponding $\sigma$. We write $\mathfrak{k}^{\sigma}=\mathfrak{k}^{(c, d)}$.

In case $\sigma$ is finite, there is the following alternative, but equivalent, way to define the subspace $\mathfrak{k}^{\sigma}$. Let $J^{\sigma} \in \operatorname{End}(V)$ be the $n \times n$ matrix with complex coefficients defined by:

$$
J^{\sigma}:=\operatorname{diag}\left(q^{\sigma}\left(q^{-\sigma}-q^{\sigma}\right), 1, \ldots, 1,0\right)-q^{\sigma} e_{1 n}-q^{\sigma} e_{n 1} .
$$

Let $M^{\sigma} \in \operatorname{End}(V) \otimes \mathcal{U}_{q}$ be the $n \times n$ matrix with coefficients in $\mathcal{U}_{q}$ defined by $M^{\sigma}:=L^{+} J^{\sigma}-J^{\sigma} L^{-}$. Then $\mathfrak{k}^{\sigma}$ is spanned by the coefficients $M_{i j}^{\sigma}$ of the matrix $M^{\sigma}$.

In the limit $q \rightarrow 1$, the subspaces $\mathfrak{k}^{\infty}$ and $\mathfrak{k}^{-\infty}$ will essentially tend to the Lie subalgebras $\mathfrak{g l}(n-1, \mathbb{C}) \oplus \mathfrak{g l}(1, \mathbb{C})$ and $\mathfrak{g l}(1, \mathbb{C}) \oplus \mathfrak{g l}(n-1, \mathbb{C})$ respectively. Moreover, the subspace $\mathfrak{k}^{0}$ will essentially tend to the Lie subalgebra $\mathfrak{k}_{\mathbb{C}}^{\prime}$ defined with respect to $(4.2)$.

Lemma 4.1 - Suppose $-\infty \leq \sigma \leq \infty$. The subspace $\mathfrak{k}^{\sigma} \subset \mathcal{U}_{q}$ is a $\tau$-invariant two-sided coideal in $\mathcal{U}_{q}$.

There are natural surjective Hopf $*$-algebra morphisms

$$
\mathcal{A}_{q} \rightarrow \mathcal{A}_{q}(U(n-1)) \otimes \mathcal{A}(U(1)), \quad \mathcal{A}_{q} \rightarrow \mathcal{A}(U(1)) \otimes \mathcal{A}_{q}(U(n-1))
$$

corresponding to the embeddings $U(n-1) \times U(1) \subset U(n)$ etc. Left or right invariance with respect to these quantum subgroups is the same as invariance with respect to the coideals $\mathfrak{k}^{\infty}$ and $\mathfrak{k}^{-\infty}$ respectively. For finite values of $\sigma$, it can be shown that there is no quantum subgroup corresponding to the coideal $\mathfrak{k}^{\sigma}$. 
Lemma 4.2 - Suppose $-\infty<\sigma<\infty$. Let $V$ be a finite-dimensional irreducible $P$-weighted $\mathcal{U}_{q}$-module. If $v \in V$ is a non-zero $\mathfrak{k}^{\sigma}$-fixed vector then the highest weight component of $v$ is non-zero.

Theorem 4.3 - Suppose $-\infty \leq \sigma \leq \infty$. For all $\lambda \in P^{+}$, the subspace $V(\lambda)_{\mathfrak{k}^{\sigma}}$ of $\mathfrak{k}^{\sigma}$-fixed vectors is at most one-dimensional. The subspace $V(\lambda)_{\mathfrak{k} \sigma}$ is one-dimensional if and only if $\lambda=l\left(\varepsilon_{1}-\varepsilon_{n}\right)$ for some $l \in \mathbb{Z}_{+}$.

In other words, the spherical representations are labelled by the same highest weights as in the classical case. We put

$$
\mathcal{B}_{q}^{(c, d)}:=\left\{a \in \mathcal{A}_{q} \mid a \cdot \mathfrak{k}^{(c, d)}=0\right\}
$$

The subspace $\mathcal{B}_{q}^{(c, d)}$ is a $*$-subalgebra and right coideal in $\mathcal{A}_{q}$, and it is invariant under the left action of $\mathcal{U}_{q}$ on $\mathcal{A}_{q}$. Vaksman and Korogodsky [VK] defined the $\mathcal{A}_{q}$-comodule algebra $\mathcal{B}_{q}^{(c, d)}$ (arbitrary values of $c, d$ ) by means of a $q$-analogue of the Hopf fibration $S^{2 n-1} \rightarrow \mathbb{C P}^{n-1}$. It can be shown that the comodule algebras $\mathcal{B}_{q}^{\sigma}=\mathcal{B}_{q}^{(c, d)}$ are non-isomorphic for different values of $\sigma$.

Theorem 4.4 - Suppose $c, d \geq 0$ such that $(c, d) \neq(0,0)$. The irreducible decomposition of $\mathcal{B}_{q}^{(c, d)}$ as a right $\mathcal{A}_{q}$-comodule resp. left $\mathcal{U}_{q}$-module is given by:

$$
\mathcal{B}_{q}^{(c, d)}=\bigoplus_{l \in \mathbb{Z}_{+}} V\left(l\left(\varepsilon_{1}-\varepsilon_{n}\right)\right)
$$

where the isotypical subspace of type $V\left(l\left(\varepsilon_{1}-\varepsilon_{n}\right)\right)$ is equal to the intersection of $\mathcal{B}_{q}^{(c, d)}$ and $W(\lambda)$.

We put

$$
z_{i j}:=d t_{1 i}^{*} t_{1 j}+c t_{n i}^{*} t_{n j}+\sqrt{c d} t_{n i}^{*} t_{1 j}+\sqrt{c d} t_{1 i}^{*} t_{n j} \in \mathcal{A}_{q} \quad(1 \leq i, j \leq n) .
$$

Proposition 4.5 - Suppose $c, d \geq 0$ such that $(c, d) \neq(0,0)$. The $z_{i j}(1 \leq$ $i, j \leq n)$ are right $\mathfrak{k}^{(c, d)}$-invariant and generate the subalgebra $\mathcal{B}_{q}^{(c, d)}$. They satisfy $z_{i j}^{*}=z_{j i}$.

Let $V^{*}$ denote the contragredient of the vector representation $V$. It has highest weight $-\varepsilon_{n}$. Let $\left(v_{i}^{*}\right)$ denote the dual basis of $V^{*}$. The tensor product representation $V^{*} \otimes V$ has the irreducible decomposition

$$
V^{*} \otimes V \cong V(0) \oplus V\left(\varepsilon_{1}-\varepsilon_{n}\right)
$$

The subspace of $\mathfrak{k}^{(c, d)}$-fixed vectors in $V^{*} \otimes V$ is two-dimensional and spanned by the two elements

$$
\sum_{k} q^{2(n-k)} v_{k}^{*} \otimes v_{k}, \quad \sqrt{c d} v_{1}^{*} \otimes v_{n}+\sqrt{c d} v_{n}^{*} \otimes v_{1}+q d v_{1}^{*} \otimes v_{1}+q^{-1} c v_{n}^{*} \otimes v_{n}
$$


Let us fix parameters $-\infty \leq \sigma, \tau \leq \infty$. We put

$$
\mathcal{H}^{(\sigma, \tau)}:=\left\{a \in \mathcal{A}_{q} \mid \mathfrak{k}^{\sigma} \cdot a=0 \text { and } a \cdot \mathfrak{k}^{\tau}=0\right\},
$$

and call it the space of $(\sigma, \tau)$-biinvariant functions. It actually is a $*$-subalgebra of $\mathcal{A}_{q}$. If we put $\mathcal{H}^{(\sigma, \tau)}(\lambda):=\mathcal{H}^{(\sigma, \tau)} \cap W(\lambda)$, then

$$
\mathcal{H}^{(\sigma, \tau)}=\bigoplus_{l \in \mathbb{Z}_{+}} \mathcal{H}^{(\sigma, \tau)}\left(l\left(\varepsilon_{1}-\varepsilon_{n}\right)\right) .
$$

Each of the spaces $\mathcal{H}^{(\sigma, \tau)}\left(l\left(\varepsilon_{1}-\varepsilon_{n}\right)\right)$ is one-dimensional. A $(\sigma, \tau)$-spherical function is by definition a non-zero element of $\mathcal{H}^{(\sigma, \tau)}\left(l\left(\varepsilon_{1}-\varepsilon_{n}\right)\right)\left(l \in \mathbb{Z}_{+}\right)$.

Let us now suppose that $\sigma, \tau$ are finite. The direct sum $\mathcal{H}^{(\sigma, \tau)}(0) \oplus \mathcal{H}^{(\sigma, \tau)}\left(\varepsilon_{1}-\varepsilon_{n}\right)$ is spanned by the unit element $1 \in \mathcal{A}_{q}$ and the element (cf. (4.6))

$$
x^{(\sigma, \tau)}:=\frac{1}{2}\left(z_{1 n}+z_{n 1}+q^{\sigma+1} z_{11}+q^{-\sigma-1} z_{n n}-\left(q^{\sigma+\tau+1}+q^{-\sigma-\tau-1}\right)\right) \in \mathcal{A}_{q} .
$$

Here (cf. (4.5))

$$
z_{i j}:=q^{\tau} t_{1 i}^{*} t_{1 j}+q^{-\tau} t_{n i}^{*} t_{n j}+t_{n i}^{*} t_{1 j}+t_{1 i}^{*} t_{n j} \in \mathcal{A}_{q} \quad(1 \leq i, j \leq n) .
$$

Note that $\left(x^{(\sigma, \tau)}\right)^{*}=x^{(\sigma, \tau)}$.

Lemma 4.6 - Let $\sigma, \tau$ be finite. Under the restriction mapping ${ }_{\mid \mathbb{T}}: \mathcal{A}_{q} \longrightarrow \mathcal{A}(\mathbb{T})$ we have:

$$
z_{11} \mapsto q^{\tau}, \quad z_{n n} \mapsto q^{-\tau}, \quad z_{1 n} \mapsto z_{1}^{-1} z_{n}, \quad z_{n 1} \mapsto z_{1} z_{n}^{-1},
$$

and all the other $z_{i j}$ are mapped onto 0 . In particular, the image of $x^{(\sigma, \tau)}$ equals $\frac{1}{2}\left(z_{1} z_{n}^{-1}+z_{1}^{-1} z_{n}\right)$.

Theorem 4.7 - Let $\sigma, \tau$ be finite. We put $z:=z_{1} z_{n}^{-1} \in \mathcal{A}(\mathbb{T})$. The restriction of the mapping ${ }_{\mid \mathbb{T}}$ to $\mathcal{H}^{(\sigma, \tau)} \subset \mathcal{A}_{q}$ is an injective $*$-algebra homomorphism onto the polynomial algebra $\mathcal{H}_{\mid \mathbb{T}}^{(\sigma, \tau)}:=\mathbb{C}\left[\frac{1}{2}\left(z+z^{-1}\right)\right] \subset \mathcal{A}(\mathbb{T})$. Hence, the algebra $\mathcal{H}^{(\sigma, \tau)}=$ $\mathbb{C}\left[x^{(\sigma, \tau)}\right]$ is commutative.

For every $l \in \mathbb{Z}_{+}$, let us fix a non-zero $\varphi_{l} \in \mathcal{H}^{(\sigma, \tau)}\left(l\left(\varepsilon_{1}-\varepsilon_{n}\right)\right)$. The $(\sigma, \tau)$ spherical function $\varphi_{l}$ is uniquely determined up to multiplication by a scalar. It can be expressed as a polynomial of degree $l$ in $x^{(\sigma, \tau)}$. In order to identify these polynomials, we study the action of the following Casimir operator on $\mathcal{H}^{(\sigma, \tau)}$ (cf. $[\mathrm{RTF}],[\mathrm{N}])$ :

$$
C:=\sum_{i j} q^{2(n-i)} L_{i j}^{+} S\left(L_{i j}^{-}\right) \in \mathcal{U}_{q}
$$

The element $C$ acts as a scalar on each subspace $\mathcal{H}^{(\sigma, \tau)}\left(l\left(\varepsilon_{1}-\varepsilon_{n}\right)\right)\left(l \in \mathbb{Z}_{+}\right)$. The corresponding eigenvalue is

$$
q^{2(l+n-1)}+q^{-2 l}+\sum_{1<i<n} q^{2(n-i)}
$$


As in (3.3), we can consider the radial part $D: \mathcal{H}_{\mid \mathbb{T}}^{(\sigma, \tau)} \rightarrow \mathcal{H}_{\mid \mathbb{T}}^{(\sigma, \tau)}$ of the Casimir operator $C$. Let us define a linear operator $T_{q, z}: \mathbb{C}\left[z, z^{-1}\right] \rightarrow \mathbb{C}\left[z, z^{-1}\right]$ by putting $T_{q, z} f(z):=f(q z)$. We use the following notation:

$$
A(z ; q):=\frac{(1-a z)(1-b z)(1-c z)(1-d z)}{\left(1-z^{2}\right)\left(1-q z^{2}\right)} \quad(a, b, c, d \in \mathbb{C}) .
$$

Theorem 4.8 - Let $\sigma, \tau$ be finite. The radial part $D: \mathcal{H}_{\mid \mathbb{T}}^{(\sigma, \tau)} \rightarrow \mathcal{H}_{\mid \mathbb{T}}^{(\sigma, \tau)}$ of the Casimir operator $C$ is equal to the following second-order $q$-difference operator:

$$
D=A\left(z ; q^{2}\right)\left(T_{q^{2}, z}-\mathrm{id}\right)+A\left(z^{-1} ; q^{2}\right)\left(T_{q^{-2}, z}-\mathrm{id}\right)+\frac{1-q^{2 n}}{1-q^{2}} \cdot \mathrm{id},
$$

with parameters $a, b, c, d$ given by

$$
a=-q^{\sigma+\tau+1}, \quad b=-q^{-\sigma-\tau+1}, \quad c=q^{\sigma-\tau+1}, \quad d=q^{-\sigma+\tau+2(n-2)+1} .
$$

For $n=2$ this result was essentially proved in Koornwinder [K4, Lemma 5.2].

Let us recall the definition of Askey-Wilson polynomials (cf. [AW]). The $q$-shifted factorials are defined as

$$
(a ; q)_{n}:=\prod_{k=0}^{n-1}\left(1-a q^{k}\right),\left(a_{1}, \ldots, a_{s} ; q\right)_{n}:=\prod_{j=1}^{s}\left(a_{j} ; q\right)_{n},(a ; q)_{\infty}:=\lim _{n \rightarrow \infty}(a ; q)_{n},
$$

and the q-hypergeometric series (cf. [GR]) as

$$
{ }_{s+1} \varphi_{s}\left[\begin{array}{c}
a_{1}, \ldots, a_{s+1} \\
b_{1}, \ldots, b_{s}
\end{array} ; q, z\right]:=\sum_{k=0}^{\infty} \frac{\left(a_{1}, \ldots, a_{s+1} ; q\right)_{k} z^{k}}{\left(b_{1}, \ldots, b_{s} ; q\right)_{k}(q ; q)_{k}} .
$$

Askey-Wilson polynomials are defined as the polynomials in $\cos (\theta)$ given by

$$
\begin{aligned}
& p_{n}(\cos (\theta) ; a, b, c, d \mid q):= \\
& \quad a^{-n}(a b, a c, a d ; q)_{n} \cdot{ }_{4} \varphi_{3}\left[\begin{array}{c}
q^{-n}, q^{n-1} a b c d, a e^{i \theta}, a e^{-i \theta} \\
a b, a c, a d
\end{array} ; q, q\right] .
\end{aligned}
$$

They are symmetric in the parameters $a, b, c, d$. Suppose that $a, b, c, d$ are real, or if complex, appear in complex conjugate pairs, and that $|a|,|b|,|c|,|d| \leq 1$ such that the pairwise products of $a, b, c, d$ are not $\geq 1$. Then the Askey-Wilson polynomials $p_{n}$ satisfy the orthogonality relations (cf. $\left.\left.[\mathrm{AW}]\right)\right)$

$$
\int_{0}^{2 \pi}\left(p_{n} p_{m}\right)(\cos \theta ; a, b, c, d \mid q)\left|\frac{\left(e^{2 i \theta} ; q\right)_{\infty}}{\left(a e^{i \theta}, b e^{i \theta}, c e^{i \theta}, d e^{i \theta} ; q\right)_{\infty}}\right|^{2} \mathrm{~d} \theta=0, n \neq m .
$$

If the condition $|a|,|b|,|c|,|d| \leq 1$ is relaxed, finitely many discrete terms will appear in the orthogonality relation (4.12).

Askey-Wilson polynomials can also be characterized as the eigenfunctions of a certain second-order $q$-difference operator. To be more precise, if we write the ${ }_{4} \varphi_{3^{-}}$ factor in (4.11) as $r_{n}(\cos (\theta))$ and put $P_{n}(z):=r_{n}\left(\frac{1}{2}\left(z+z^{-1}\right)\right)$, then the Laurent polynomials $P_{n}(z)$ in the variable $z$ satisfy the following second-order $q$-difference equation:

$$
\begin{aligned}
A(z ; q)\left(P_{n}(q z)-P_{n}(z)\right) & +A\left(z^{-1} ; q\right)\left(P_{n}\left(q^{-1} z\right)-P_{n}(z)\right)= \\
& =-\left(1-q^{-n}\right)\left(1-q^{n-1} a b c d\right) P_{n}(z),
\end{aligned}
$$

where $A(z ; q)$ is defined in (4.10). Any polynomial $f\left(e^{i \theta}\right)$ of degree $\leq n$ in $\cos \theta$ satisfying (4.13) with $z=e^{i \theta}$ is a constant multiple of $P_{n}\left(e^{i \theta}\right)$.

Comparing (4.13) with [4.8] we conclude: 
Theorem 4.9 - Let $\sigma, \tau$ be finite. The $(\sigma, \tau)$-spherical functions in $\mathcal{H}^{(\sigma, \tau)}\left(l\left(\varepsilon_{1}-\right.\right.$ $\left.\left.\varepsilon_{n}\right)\right)\left(l \in \mathbb{Z}_{+}\right)$are spanned by

$$
p_{l}\left(x^{(\sigma, \tau)} ;-q^{\sigma+\tau+1},-q^{-\sigma-\tau+1}, q^{\sigma-\tau+1}, q^{-\sigma+\tau+2(n-2)+1} \mid q^{2}\right),
$$

where $p_{l}$ is an Askey-Wilson polynomial.

For $n=2$ this result was essentially proved by Koornwinder [K4, Theorem 5.2].

Using [4.9] and a suitable limit argument (cf. [K4, Prop. 6.1, 6.3]), one can identify the spherical functions corresponding to the cases when either $\sigma$ or $\tau$ is infinite. They are expressed as so-called little and big q-Jacobi polynomials (cf. $[\mathrm{AA}]$ ). These are orthogonal polynomials with respect to an measure supported on an infinite discrete set. For more details see [DN].

\section{Higher rank quantum symmetric spaces}

We refer to Table I for an exhaustive list (up to local isomorphism) of the classical irreducible compact Riemannian symmetric spaces of type I (cf. [H1]). The column labelled CC contains the type designation as used by É. Cartan in his classification. In the last column we give the multiplicities of the short, medium-sized, and long restricted roots (in this order). Here we consider $C_{l}$ as a root system of type $B C$, the short roots having multiplicity zero.

Table I: Classical irreducible compact symmetric spaces

\begin{tabular}{lllllll}
\hline no. & CC & $G$ & $K$ & $l$ & $\Sigma$ & $m_{\alpha}$ \\
\hline 1 & AI & $S U(n)$ & $S O(n)$ & $l=n-1$ & $A_{l}$ & 1 \\
2 & AII & $S U(2 n)$ & $S p(n)$ & $l=n-1$ & $A_{l}$ & 4 \\
3 & AIII & $U(n)$ & $U(l) \times U(n-l)$ & $l \leq\left[\frac{n}{2}\right]$ & $B C_{l}$ & $2(n-2 l), 2,1$ \\
4 & BI & $S O(2 n+1)$ & $S O(l) \times S O(2 n+1-l)$ & $l \leq n$ & $B_{l}$ & $1,2 n+1-2 l$ \\
5 & CI & $S p(n)$ & $U(n)$ & $l=n$ & $C_{l}$ & $0,1,1$ \\
6 & CII & $S p(n)$ & $S p(l) \times S p(n-l)$ & $l \leq\left[\frac{n}{2}\right]$ & $B C_{l}$ & $4(n-2 l), 4,3$ \\
7 & DI & $S O(2 n)$ & $S O(l) \times S O(2 n-l)$ & $l<n$ & $B_{l}$ & $2 n-2 l, 1$ \\
8 & DI & $S O(2 n)$ & $S O(l) \times S O(2 n-l)$ & $l=n$ & $D_{l}$ & 1 \\
9 & DIII & $S O(2 n)$ & $U(n)$ & $n=2 l$ & $C_{l}$ & $0,4,1$ \\
10 & DIII & $S O(2 n)$ & $U(n)$ & $n=2 l+1$ & $B C_{l}$ & $4,4,1$ \\
& & & & &
\end{tabular}

Let $(G, K)$ be any of the symmetric pairs listed in Table I. Suppose we have a twosided coideal $\mathfrak{k}_{q} \subset \mathcal{U}_{q}$ which, in some suitable sense, has $\mathfrak{k}_{\mathbb{C}} \subset \mathfrak{g}_{\mathbb{C}}$ as its classical limit. From the results in section 4 we may draw the conclusion that the relative position of $\mathfrak{k}_{\mathbb{C}}$ with respect to the standard Cartan subalgebra $\mathfrak{h}_{\mathbb{C}} \subset \mathfrak{g}_{\mathbb{C}}$ is of considerable importance in the analysis of the quantum symmetric space corresponding to $\mathfrak{k}_{q}$ and its zonal spherical functions. We distinguish three different cases.

The first case arises when $\mathfrak{h}_{\mathbb{C}}$ is $\theta$-invariant and $\mathfrak{k}_{\mathbb{C}}$ such that the intersection $\mathfrak{h}_{\mathbb{C}} \cap \mathfrak{p}_{\mathbb{C}}$ is maximal abelian in $\mathfrak{p}_{\mathbb{C}}$, hence equal to $\mathfrak{a}_{\mathbb{C}}$. We shall term this the maximally split case. In this situation one can reasonably expect the restriction of $\mathfrak{k}_{q}$-biinvariant functions to the diagonal subgroup $\mathbb{T}$ to be injective and the spherical 
functions to be multivariable orthogonal polynomials with respect to a continuous measure.

Actually, so far, Noumi and Sugitani $[\mathrm{N}],[\mathrm{NS} 1],[\mathrm{NS} 2],[\mathrm{Su}]$ have constructed a maximally split coideal $\mathfrak{k}_{q} \subset \mathcal{U}_{q}$ for all symmetric pairs $(G, K)$ listed in Table $\mathrm{I}$, and analysed the corresponding quantum symmetric spaces and their spherical functions. We shall give a very brief account of their results for the symmetric pairs (1), (2), and (4)-(10).

These symmetric pairs have in common that the "lowest" non-trivial spherical representation occurs in the irreducible decomposition of the tensor product $V \otimes V$ of the vector representation $V$ with itself. In the quantum case, the existence of $\mathfrak{k}_{q^{-}}$ fixed vectors inside $V \otimes V$ is controlled by the so-called reflection equation (cf. [Ku]). Suppose we have an invertible complex $N \times N$ matrix $J$ (recall that $N:=\operatorname{dim}(V)$ ). Define a matrix $M \in \operatorname{End}(V) \otimes \mathcal{U}_{q}$ with coefficients in $\mathcal{U}_{q}$ by putting

$$
M:=L^{+}-J S\left(L^{-}\right)^{t} J^{-1},
$$

and define $\mathfrak{k}_{q} \subset \mathcal{U}_{q}$ as the subspace spanned by the coefficients $M_{i j}$ of $M$. Then $\mathfrak{k}_{q}$ will be a two-sided coideal in $\mathcal{U}_{q}$. Put $w_{J}=\sum_{i j} v_{i} \otimes J_{i j} v_{j} \in V \otimes V$. Then it can be shown that $\mathfrak{k}_{q} \cdot w_{J}=0$ if and only if the matrix $J$ satisfies the following reflection equation:

$$
R J_{1} R^{t_{1}} J_{2}=J_{2} R^{t_{1}} J_{1} R,
$$

where ${ }^{t_{1}}$ denotes transposition in the first tensor component, and $J_{1}:=J \otimes$ id etc. are Kronecker matrix products.

For every symmetric pair $(G, K)$, Noumi and Sugitani give a constant (i.e. involving no parameters besides $q$ ) matrix $J$ satisfying the reflection equation and such that the corresponding $\mathfrak{k}_{q}$ is invariant under $\tau=* \circ S$. Let $\rho \in P$ be the half sum of the positive roots. The element $q^{\rho} \in \mathcal{U}_{q}(\mathfrak{h})$ acts on $V$ as a diagonal matrix written $\operatorname{diag}\left(q^{\rho_{1}}, \ldots, q^{\rho_{N}}\right)$. Then the matrix $J$ is given by:

$$
\begin{aligned}
& J=\operatorname{diag}\left(q^{\rho_{1}}, \ldots, q^{\rho_{N}}\right) \quad \text { for } \quad(1),(5),(8), \\
& J=J_{0} \operatorname{diag}\left(q^{\rho_{1}}, \ldots, q^{\rho_{N}}\right) \text { for } \quad(2),(6)(n=2 l),(9),(10),
\end{aligned}
$$

where $J_{0}:=\sum_{k=1}^{N}\left(-e_{2 k, 2 k-1}+e_{2 k-1,2 k}\right)$. For the definition of $J$ in the remaining cases see [NS2], [Su]. In all cases, the pairs $\left(\mathcal{U}_{q}, \mathfrak{k}_{q}\right)$ are quantum Gelfand pairs. The spherical representations are labelled by the same subset $P_{K}^{+}$of highest weights as in the classical case.

Noumi and Sugitani next study the $*$-subalgebra $\mathcal{H} \subset \mathcal{A}_{q}$ of $\mathfrak{k}_{q}$-biinvariant functions. To be precise, in the cases (4)-(10) they consider functions which are left-invariant with respect to $\mathfrak{k}_{q}$ and right invariant with respect to $\overline{\mathfrak{k}_{q}}$, where $\bar{u}:=q^{\rho} u^{*} q^{-\rho}$ is a modified $*$-operation on $\mathcal{U}_{q}$. They show that the mapping ${ }_{\mid \mathbb{T}}: \mathcal{H} \rightarrow \mathcal{A}(\mathbb{T})$ is injective and describe the image $\mathcal{H}_{\mid \mathbb{T}} \subset \mathcal{A}(\mathbb{T})$ in terms of certain explicitly defined elements $x_{1}, \ldots, x_{l} \in \mathcal{A}(\mathbb{T})$. More precisely, they show this subalgebra is isomorphic with $\mathcal{A}(\Sigma)^{W}$ under the correspondence $x_{i} \mapsto e^{\varepsilon_{i}}$.

Let us recall the definition of Macdonald's symmetric orthogonal polynomials (cf. [M1], [M2], [M3]) and Koornwinder's $B C$-type Askey-Wilson polynomials (cf. [K3]). In Macdonald's case, let $\Sigma$ be one of the root systems $A_{l}, B_{l}, C_{l}, D_{l}$. In Koornwinder's case we put $\Sigma=B C_{l}$. We freely use the notation introduced in section 1 . 
We first treat Macdonald's case. Let $k: \alpha \mapsto k_{\alpha}$ be a (non-negative) multiplicity function. Assume the standard normalization of the $W$-invariant inner product. We put $u_{\alpha}=1$ (type $A$ ), $u_{\alpha}=\frac{\langle\alpha, \alpha\rangle}{2}$ (type $\left.B, C, D\right)$ for all $\alpha \in \Sigma$. Set $q_{\alpha}=q^{u_{\alpha}}$, $t_{\alpha}=q^{k_{\alpha}}(\alpha \in \Sigma)$. We define an inner product on functions on the torus $T$ by putting:

$$
\Delta^{+}:=\prod_{\alpha \in \Sigma^{+}} \frac{\left(e^{\alpha} ; q_{\alpha}\right)_{\infty}}{\left(t_{\alpha} e^{\alpha} ; q_{\alpha}\right)_{\infty}}, \quad \Delta:=\Delta^{+} \overline{\Delta^{+}}, \quad\langle f, g\rangle_{k}:=\int_{T} f(x) \overline{g(x)} \Delta(x) d x .
$$

Using this inner product instead of (1.2), one now proceeds in exactly the same way as in section 1 to obtain (cf. [M1], [M2], [M3]) Macdonald's symmetric polynomials $P_{\lambda}^{k} \in \mathcal{A}(\Sigma)^{W}\left(\lambda \in P^{+}\right)$corresponding to the pair $(\Sigma, \Sigma)$ (type $\left.A\right)$ or $\left(\Sigma, \Sigma^{\vee}\right)$ (type $B, C, D)$.

In Koornwinder's case, it is convenient to use the notation $x_{i}=e^{\varepsilon_{i}}, x^{\lambda}=e^{\lambda}$ for $\lambda \in P$. Recall that $P$ is the free $\mathbb{Z}$-span of the standard basis vectors $\varepsilon_{i} \in P$. The definition of Koornwinder's BC-type Askey-Wilson polynomials is completely analogous to Macdonald's case, except that one has to take a different weight function $\Delta^{+}$(cf. (4.12)):

$$
\Delta^{+}(x):=\prod_{k=1}^{l} \frac{\left(x_{k}^{2} ; q\right)_{\infty}}{\left(a x_{k}, b x_{k}, c x_{k}, d x_{k} ; q\right)_{\infty}} \cdot \prod_{i<j} \frac{\left(x_{i} / x_{j}, x_{i} x_{j} ; q\right)_{\infty}}{\left(t x_{i} / x_{j}, t x_{i} x_{j} ; q\right)_{\infty}}(a, b, c, d, t \in \mathbb{C}) .
$$

For $n=1$ one reobtains the Askey-Wilson polynomials defined in section 4 .

To prove that the polynomials $P_{\lambda}$ are mutually orthogonal, Macdonald [M1], [M2] and Koornwinder [K3] exhibit a self-adjoint partial $q$-difference operator $D_{\sigma}$ (depending on a minuscule weight $\sigma \in P^{+}$) on $\mathcal{A}(\Sigma)^{W}$ which is diagonalized by the $P_{\lambda}$. Here we take $\sigma=\varepsilon_{1}$. The definition of $D_{\sigma}$ then reads:

$$
\Phi_{\sigma}:=\frac{T_{\sigma} \Delta^{+}}{\Delta^{+}}, \quad D_{\sigma} f:=|W \sigma|^{-1} \sum_{w \in W}\left(w \Phi_{\sigma}\right)\left(T_{w \sigma} f-f\right), \quad f \in \mathcal{A}(\Sigma)^{W} .
$$

Here $T_{\mu}(\mu \in P)$ is the operator defined on $\mathcal{A}(\Sigma)$ by $T_{\mu} x^{\lambda}=q^{\langle\lambda, \mu\rangle} x^{\mu}(\lambda \in P)$. The operator $D_{\sigma}$ maps $A(\Sigma)^{W}$ into itself and is diagonalized by the $P_{\lambda}$. Koornwinder proves the orthogonality of the $P_{\lambda}$ under the assumption that (i) the parameters $a, b, c, d$ are real or, if complex, appear in conjugate pairs, (ii) $|a|,|b|,|c|,|d| \leq 1$, but the pairwise products of $a, b, c, d$ are not $\geq 1$, (iii) $t \in(-1,1)$. In this case, the weight function $\Delta$ is continuous on the torus $T$.

Let us return to the setting of quantum symmetric spaces. In all cases described above, Noumi and Sugitani have been able to show that the radial part $D$ of a suitable Casimir operator $C \in \mathcal{Z U}_{q}$ (cf. (4.9)) is essentially the same as the partial $q$-difference operator $D_{\sigma}$ corresponding to the restricted root system $\Sigma=\Sigma(G, K)$. The multiplicity function $k$ resp. the parameters $a, b, c, d, t$ are defined in terms of $q$ and the root multiplicities of $(G, K)$ in a way comparable to (1.4). This result allows them to prove that the zonal spherical functions $\varphi(\lambda)\left(\lambda \in P_{K}^{+}\right)$, when restricted to the diagonal subgroup $\mathbb{T}$, can be expressed as Macdonald's polynomials or Koornwinder's Askey-Wilson polynomials.

The second case of the three cases alluded to above arises when the embedding $\mathfrak{k}_{\mathbb{C}} \subset \mathfrak{g}_{\mathbb{C}}$ is induced by an embedding of Dynkin diagrams. By deleting one node in 
the Dynkin diagram of $\mathfrak{g}_{\mathbb{C}}$, one obtains the (possibly disconnected) Dynkin diagram of a semisimple complex Lie algebra $\mathfrak{g}_{\mathbb{C}}^{\prime}$. The Lie algebra $\mathfrak{k}_{\mathbb{C}}:=\mathfrak{g}_{\mathbb{C}}^{\prime} \oplus \mathbb{C}$ naturally is a Lie subalgebra of $\mathfrak{g}_{\mathbb{C}}$. The irreducible symmetric pairs $(G, K)$ that can be obtained via this procedure are exactly the irreducible Hermitian symmetric pairs ${ }^{1}$. Observe that the Hermitian spaces in Table I are those for which the root system is $B C_{l}$ and the long roots have multiplicity 1 (recall that $B_{2} \simeq C_{2}^{\vee} \simeq C_{2}$ is considered to be of type $B C$ ).

Since the definition of $\mathcal{U}_{q}\left(\mathfrak{g}_{\mathbb{C}}\right)$ is given in terms of generators indexed by simple roots and relations depending only on the Cartan matrix of $\mathfrak{g}_{\mathbb{C}}$, there will be a natural embedding of Hopf $*$-algebras $\mathcal{U}_{q}\left(\mathfrak{k}_{\mathbb{C}}\right) \subset \mathcal{U}_{q}\left(\mathfrak{g}_{\mathbb{C}}\right)$ and a corresponding surjective Hopf $*$-algebra morphism $\mathcal{A}_{q}(G) \rightarrow \mathcal{A}_{q}(K)$. From here, it is easy to define a suitable coideal $\mathfrak{k}_{q} \subset \mathcal{U}_{q}\left(\mathfrak{g}_{\mathbb{C}}\right)$ (cf. section 3 ). We shall call this the regular embedded case. It is quite opposite to the maximally split case, since now the intersection $\mathfrak{h}_{\mathbb{C}} \cap \mathfrak{k}_{\mathbb{C}}$ is maximal abelian in $\mathfrak{k}_{\mathbb{C}}$. For this reason, the restriction of $\mathfrak{k}_{q}$-biinvariant functions to the diagonal subgroup $\mathbb{T}$ will not be injective. Therefore, the method proposed in section 3 does not work. To the author's knowledge, the spherical functions in these cases have not yet been studied, except in rank one (cf. [VS], [Mas], [K1], $[\mathrm{NYM}])$. It is likely, though, that the spherical functions in this case will be some kind of polynomials orthogonal with respect to a discrete orthogonality measure. Until recently, not much was known about multivariable orthogonal polynomials with a discrete measure in connection with root systems (however, see [St]).

The third case is a kind of interpolation between the regular embedded and maximally split cases. It arises when the Cartan algebra $\mathfrak{h}_{\mathbb{C}}$ is not necessarily invariant under the involution $\theta$, but its projection onto $\mathfrak{p}_{\mathbb{C}}$ along the decomposition $\mathfrak{g}_{\mathbb{C}}=\mathfrak{k}_{\mathbb{C}} \oplus \mathfrak{p}_{\mathbb{C}}$ still is maximal abelian in $\mathfrak{p}_{\mathbb{C}}$. In this case, the restriction of $\mathfrak{k}_{q^{-}}$ biinvariant functions to $\mathbb{T}$ is still likely to be an injective operation.

With this terminology, the coideal $\mathfrak{k}^{\sigma}$ defined in section 4 is regular embedded $(\sigma= \pm \infty)$, maximally split $(\sigma=0)$, and interpolated ( $\sigma$ finite and non-zero). In the last case, the spherical functions are orthogonal with respect to a mixed continuous and discrete orthogonality measure.

Very recently, Noumi, Sugitani and the author constructed a one-parameter family of coideals $\mathfrak{k}^{\sigma} \subset \mathcal{U}_{q}(\mathfrak{g l}(n, \mathbb{C}))(-\infty<\sigma<\infty)$ defining a quantum analogue of the symmetric space $U(n) / U(l) \times U(n-l)\left(l \leq\left[\frac{n}{2}\right]\right)$. This symmetric space is different from the others in the sense that the "lowest" spherical representation occurs in the decomposition of the tensor product $V \otimes V^{*}$. In this case, the reflection equation reads

$$
R^{+} J_{2} R_{21}^{-} J_{1}=J_{1} R^{-} J_{2} R_{21}^{+},
$$

with $R_{21}^{ \pm}:=P R^{ \pm} P$. The coideal $\mathfrak{k}^{\sigma}$ is by definition spanned by the coefficients of the matrix $M:=L^{+} J^{\sigma}-J^{\sigma} L^{-}$, where

$$
J^{\sigma}:=\sum_{1 \leq k \leq l} q^{\sigma}\left(q^{-\sigma}-q^{\sigma}\right) e_{k k}+\sum_{l<k<l^{\prime}} e_{k k}-\sum_{k \leq l \text { or } k \geq l^{\prime}} q^{\sigma} e_{k k^{\prime}},
$$

and $k^{\prime}:=n+1-k(1 \leq k \leq n)$. This matrix $J^{\sigma}$ is a solution of the reflection equation (5.5). For $l=1$ we reobtain (4.4). The radial part of the Casimir operator (4.9) in this case is essentially the same as Koornwinder's $q$-difference operator $D_{\varepsilon_{1}}$, the parameters $a, b, c, d, t$ depending on two continuous parameters $\sigma, \tau$, and two discrete parameters $n, l$ (besides $q$ ). For details see the forthcoming paper [NDS].

\footnotetext{
${ }^{1}$ The author owes these observations to Prof. Tom H. Koornwinder.
} 


\section{REFERENCES}

[AA] G.E. Andrews, R. Askey, Classical orthogonal polynomials, in: "Polynômes Orthogonaux et Applications", ed. C. Brzezinski, A. Draux, P. Magnus, P. Maroni, A. Ronveaux, Lecture Notes in Mathematics 1171, Springer-Verlag, 1985, pp. 36-62.

[AW] R. Askey, J. Wilson, Some basic hypergeometric orthogonal polynomials that generalize Jacobi polynomials, Mem. Amer. Math. Soc. 54 (1985), no. 319.

[Ch] W. Chin, I.M. Musson, The coradical filtration for quantized universal enveloping algebras, preprint.

[DK1] M.S. Dijkhuizen, T.H. Koornwinder, Quantum homogeneous spaces, duality and quantum 2-spheres, Geom. Dedicata 52 (1994), 291-315.

[DK2] - CQG algebras: a direct algebraic approach to compact quantum groups, Lett. Math. Phys. 32 (1994), 315-330.

[DN] M.S. Dijkhuizen, M. Noumi, A family of quantum projective spaces and related $q$-hypergeometric orthogonal polynomials, preprint (1995).

[Dr] V.G. Drinfel'd, Quantum groups, in: Proceedings ICM Berkeley (1986), ed. A.M. Gleason, Amer. Math. Soc., Providence, RI, 1986, pp. 798-820.

[GR] G. Gasper, M. Rahman, Basic hypergeometric series, Encyclopedia of Mathematics and Its Applications 35, Cambridge University Press, London, 1990.

[Ha] T. Hayashi, Non-existence of homomorphisms between quantum groups, Tokyo J. Math. (to appear).

[HS] G.J. Heckman, H. Schlichtkrull, Harmonic Analysis and Special Functions on Symmetric Spaces, Perspectives in Math. 16, Academic Press, 1995.

[H1] S. Helgason, Differential Geometry, Lie Groups, and Symmetric Spaces, Pure and Applied Mathematics 80, Academic Press, 1978.

[H2] Groups and Geometric Analysis, Pure and Applied Mathematics 113, Academic Press, 1984.

[J1] M. Jimbo, A q-difference analogue of $U(\mathfrak{g})$ and the Yang-Baxter equation, Lett. Math. Phys. 10 (1985), 63-69.

[J2] , Quantum R-matrix for the generalized Toda system, Comm. Math. Phys. 102 (1986), 537-547.

[J3] , A q-analogue of $U(\mathfrak{g l}(n))$, Hecke algebra and the Yang-Baxter equation, Lett. Math. Phys. 11 (1986), 247-252.

[K1] T.H. Koornwinder, Representations of the twisted $S U(2)$ quantum group and some qhypergeometric polynomials, Proc. Kon. Ned. Akad. Wet. Series A 92 (1989), 97-117.

[K2] - Orthogonal polynomials in connection with quantum groups, in: "Orthogonal polynomials: Theory and Practice", ed. P. Nevai, NATO-ASI Series C 294, Kluwer, Norwell, MA, 1990, pp. 257-292.

[K3] - Askey-Wilson polynomials for root systems of type BC, in: "Hypergeometric functions on domains of positivity, Jack polynomials, and applications", ed. D.S.P. Richards Contemp. Math. 138, Amer. Math. Soc., Providence, RI, 1992, pp. 189-204.

[K4] Askey-Wilson polynomials as zonal spherical functions on the $S U(2)$ quantum group, SIAM J. Math. Anal. 24 (1993), no. 3, 795-813.

[VK] L.I. Korogodsky, L.L. Vaksman, Quantum G-spaces and Heisenberg algebra, in: "Quantum Groups", ed. P.P. Kulish, Lecture Notes in Math. 1510, Springer-Verlag, 1992, pp. 5666.

[Ku] P.P. Kulish, Quantum groups and quantum algebras as symmetries of dynamical systems, preprint YITP/K-959 (1991).

[Lz] G. Lusztig, Quantum deformations of certain simple modules over enveloping algebras, Adv. Math. 70 (1988), 237-249.

[M1] I.G. Macdonald, A new class of symmetric functions, Séminaire Lotharingien de Combinatoire, ed. L. Cerlienco, D. Foata, Publication I.R.M.A. 372/S-20, Strasbourg, 1988, pp. 131-171.

[M2] - Orthogonal polynomials associated with root systems, preprint (1988).

[M3] - Affine Hecke algebras and orthogonal polynomials, Séminaire Bourbaki 47 (199495), no. 797, 1-18.

[Mas] T. Masuda, K. Mimachi, Y. Nakagami, M. Noumi, K. Ueno, Representations of quantum groups and a q-analogue of orthogonal polynomials, C. R. Acad. Sci. Paris Sér. I Math. 307 (1988), 559-564. 
[N] M. Noumi, Macdonald's symmetric polynomials as zonal spherical functions on some quantum homogeneous spaces, Adv. Math. (to appear).

[NDS] M. Noumi, M.S. Dijkhuizen, T. Sugitani, Multivariable Askey-Wilson polynomials and quantum complex Grassmannians, in: Proceedings of a Workshop on $q$-Series, Special Functions and Related Topics, Toronto (1995), Fields Inst. Comm. (to appear).

[NS1] M. Noumi, T. Sugitani, Quantum symmetric spaces and related q-orthogonal polynomials, in: "Group Theoretical Methods in Physics", Proceedings XX ICGTMP, Toyonaka (Japan), 1994, ed. A. Arima et al., World Scientific, Singapore, 1995, pp. 28-40.

[NS2] , in preparation.

[NYM] M. Noumi, H. Yamada, K. Mimachi, Finite-dimensional representations of the quantum group $G L_{q}(n, \mathbb{C})$ and the zonal spherical functions on $U_{q}(n-1) \backslash U_{q}(n)$, Japanese J. Math. 19 (1993), no. 1, 31-80.

[Po] P. Podleś, Quantum spheres, Lett. Math. Phys. 14 (1987), 193-202.

[RTF] N. Reshetikhin, L.D. Faddeev, L.A. Takhtadjan, Quantization of Lie groups and Lie algebras, Leningrad Math. J. 1 (1990), 193-225.

[R] M. Rosso, Finite-dimensional representations of the quantum analog of a complex simple Lie algebra, Comm. Math. Phys. 117 (1988), 581-593.

[St] J. Stokman, Multivariable big and little q-Jacobi polynomials, Mathematical Preprint Series 95-16, University of Amsterdam (1995).

[Su] T. Sugitani, in preparation.

[UT] K. Ueno, T. Takebayashi, Zonal spherical functions on quantum symmetric spaces and Macdonald's symmetric polynomials, in: "Quantum Groups", ed. P.P. Kulish, Lecture Notes in Math. 1510, Springer-Verlag, 1992, pp. 142-147.

[VS] L.L. Vaksman, Y.S. Solbel'man, Algebra of functions on the quantum group SU(2), Funct. Anal. Appl. 22 (1988), 170-181.

[Wo] S.L. Woronowicz, Compact matrix pseudogroups, Comm. Math. Phys. 111 (1987), 613665. 